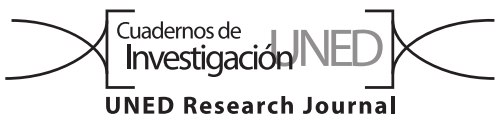

\title{
I Simposio sobre cambio climático y Biodiversidad: hacia el fortalecimiento de la resiliencia y acciones requeridas ante el cambio climático en Latinoamérica
}

\author{
Jimena Samper-Villarreal ', Andrea Vincent ${ }^{2}$, Carolina Álvarez ${ }^{3}$, \& Gustavo A. Gutiérrez-Espeleta ${ }^{2}$ \\ 1. Universidad de Costa Rica, Centro de Investigación en Ciencias del Mar y Limnología, 11501-2060 San José, Costa Rica; \\ jimena.sampervillarreal@ucr.ac.cr \\ 2. Universidad de Costa Rica, Escuela de Biología, 11501-2060 San José, Costa Rica. \\ 3. Facultad Latinoamericana de Ciencias Sociales (FLACSO), Sede Costa Rica.
}

Recibido 22-IX-2018 • Corregido 22-XII-2018 • Aceptado 14-I-2019

\begin{abstract}
I Symposium on Climate Change and Biodiversity: Towards strengthening the resilience and actions required to face Climate Change in Latin America. Climate change is a global phenomenon with irreversible consequences if timely action is not taken. In Latin America, climate change does not receive the attention it deserves in the political, academic and citizen agenda, and information and research in Spanish is limited. The I Symposium on Climate Change and Biodiversity, which took place in August 2017 in Costa Rica, sought to generate interest and discussion on possible solutions in the face of climate change in Latin America before a multidisciplinary and inter institutional audience. The Symposium was organized into four topics: 1) Impacts on ecosystems; 2) Mitigation; 3) Adaptation; and 4) Communication and education in the face of climate change. Here, we carry out a critical synthesis of the diverse subjects presented the symposium, some of which are developed in more detail in this special issue of UNED Research Journal. There is no question that climate change is real and that it is caused by human activities, which presents us with the opportunity to change our individual actions and policy decisions to reduce green-house gas emissions and increase their mitigation, as well as to adapt to the new reality this phenomenon brings.
\end{abstract}

Key words: Global warming, adaptation, mitigation, governance, environment, agroecology.
RESUMEN: El cambio climático es un fenómeno global con consecuencias irreversibles si no se toman las acciones pertinentes. En Latinoamérica, el cambio climático no recibe la atención merecida en la agenda política, académica ni ciudadana, consecuentemente, la información en español e investigaciones acerca de esta temática son limitadas. El I Simposio sobre cambio climático y Biodiversidad, realizado en agosto del año 2017, en Costa Rica, frente a una audiencia multidisciplinaria e interinstitucional, buscó generar interés y discusión sobre posibles soluciones ante el cambio climático en esta región. El simposio se organizó según cuatro ejes temáticos: 1) Impactos sobre ecosistemas, 2) Mitigación, 3) Adaptación, y 4) Comunicación y enseñanza del cambio climático. En esta publicación, desarrollamos una síntesis crítica de los diversos temas tratados en dicho simposio, algunos de los cuales se desarrollan en mayor profundidad en este número especial de UNED Research Journal. No cabe duda de que el cambio climático es real y tiene su origen en las actividades humanas, lo que implica que debemos cambiar nuestras acciones individuales y decisiones políticas para reducir la emisión e incrementar la mitigación de gases de efecto invernadero, así como para adaptarnos a la nueva realidad que este fenómeno implica.

Palabras clave: Calentamiento global, adaptación, mitigación, gobernanza, ambiente, agroecología.
Los cambios antropogénicos han afectado el sistema climático global y han ocasionado un fenómeno mundial conocido como cambio climático. La principal actividad humana que modifica el ambiente es la emisión de gases de efecto invernadero, tales como el dióxido de carbono $\left(\mathrm{CO}_{2}\right)$, óxido nitroso y metano. Estos gases provienen de la quema de combustibles fósiles, deforestación masiva, actividad ganadera, cambio de uso suelo y uso de agroquímicos, por mencionar los principales (Pachauri et al., 2014).
Debido a esta emisión de gases, la atmósfera y el océano se han calentado, esto genera el derretimiento de los casquetes polares y el crecimiento y acidificación de los océanos (Pachauri et al., 2014). Actualmente, nos encontramos en una nueva era geológica, el Antropoceno (Waters et al., 2016), y somos la primera generación que tiene plena conciencia de que el cambio climático está ocurriendo, por lo tanto, es nuestra responsabilidad actuar para detenerlo o, al menos, para disminuir su impacto al máximo tanto a nivel global como en la región latinoamericana. 
Latinoamérica es una región rica en biodiversidad, áreas protegidas y con una matriz eléctrica con fuentes renovables. Sin embargo, también es una región altamente vulnerable al cambio climático, con fuerte dependencia del agro y del turismo, amplias zonas inundables y fuertes retos socio-económicos (Galindo, de Miguel \& Ferrer, 2010). En este momento, en Latinoamérica, el cambio climático no recibe la atención merecida en la agenda política, académica ni ciudadana. Además, la información en español es limitada y se concentra en grupos selectos, principalmente, en la Academia. Resulta evidente, entonces, que las personas responsables de la política, y los diversos actores asociados al cambio climático, en la región, requieren de información adecuada para la toma de decisiones.

Con estos retos en mente, se desarrolló el / Simposio sobre cambio climático y Biodiversidad, entre el 9 y el 11 de agosto del año 2017, en San José, Costa Rica. Fue organizado por la Red Latinoamericana de Ciencias Biológicas (RELAB), la Universidad de Costa Rica (UCR) y la Academia Nacional de Ciencias de Costa Rica (ANC). El objetivo de este simposio fue generar un encuentro público de personas inmersas en la problemática del cambio climático y la biodiversidad, a nivel latinoamericano, con el fin de sensibilizar e informar a la sociedad, generar interés sobre el tema y discutir soluciones a nivel regional. Esta actividad contó con charlas magistrales, acompañadas de espacios abiertos de discusión y análisis, por parte de todas las personas participantes (Fig. 1).

En este simposio presentaron un total de 23 personas expositoras internacionales. Al evento asistieron un total de 215 personas de 14 países: Uruguay, Chile, Perú, Ecuador, Colombia, Venezuela, El Salvador, Panamá, Guatemala, México, Estados Unidos de América, Cuba, Alemania y Costa Rica, lo que permitió un diálogo sobre el cambio climático y biodiversidad. Este diálogo abarcó temáticas desde las zonas templadas del Norte y Sur del planeta, hasta los trópicos y ciudades cosmopolitas. El simposio se organizó en cuatro ejes temáticos (Fig. 2): 1) Impactos del cambio climático sobre ecosistemas, 2) Mitigación, 3) Adaptación, y 4) Comunicación y aprendizaje sobre el cambio climático.

A continuación, presentamos una síntesis crítica de los diversos temas desarrollados durante el Simposio, algunos de los cuales se desarrollan en mayor profundidad en este número especial de UNED Research Journal.

\section{IMPACTOS DEL CAMBIO CLIMÁTICO SOBRE ECOSISTEMAS TERRESTRES Y MARINOS}

El cambio climático ejerce impactos directos e indirectos sobre los ecosistemas y la biodiversidad; cada ambiente y su biota responden de manera específica a su
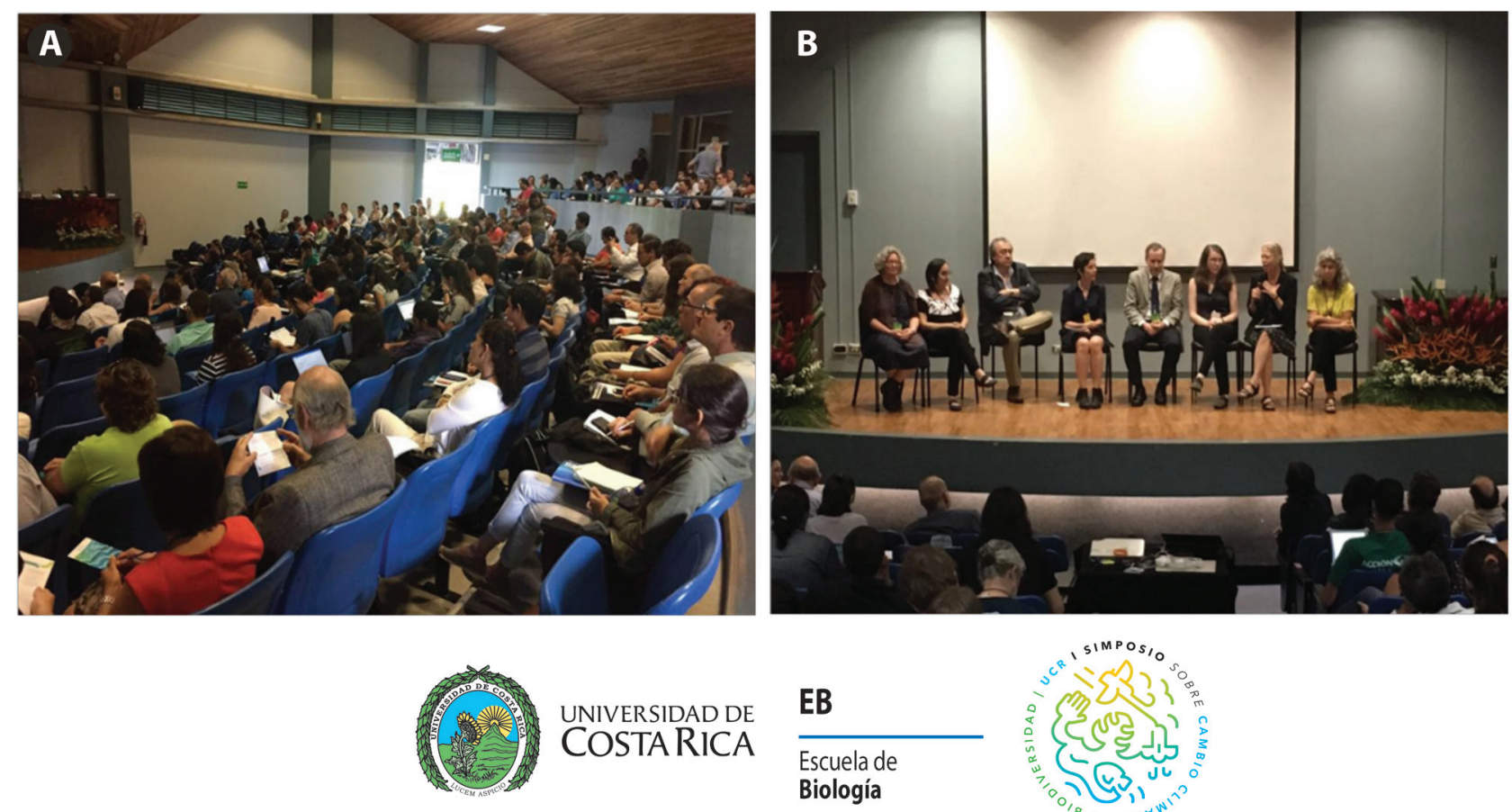

EB

Escuela de

Biología

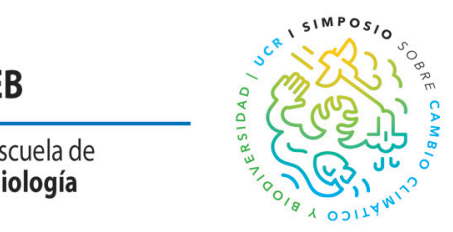

Fig. 1. Participantes (a) y uno de los paneles de personas expositoras (b) durante el I Simposio sobre cambio climático y Biodiversidad. 9 - 11 de agosto de 2017, San José, Costa Rica. 


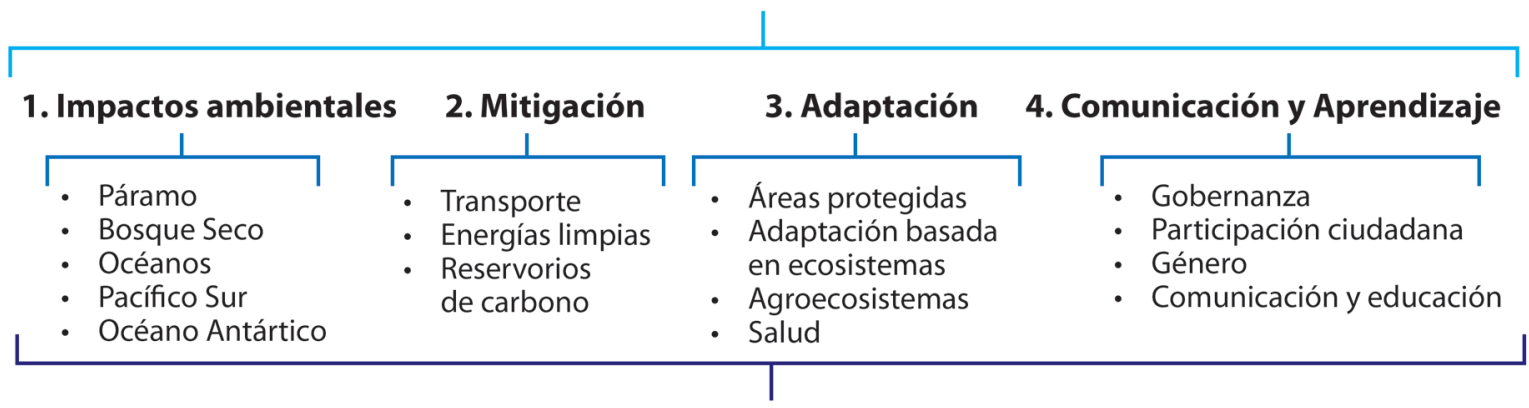

Factores a considerar para la resiliencia de Latinoamérica y acciones requeridas ante el Cambio Climático

Fig. 2. Ejes temáticos del / Simposio sobre cambio climático y Biodiversidad.

influencia. Latinoamérica es una región altamente biodiversa con un extraordinario mosaico de ecosistemas, tanto terrestres como marinos. A continuación, se detallan los principales impactos del cambio climático sobre algunos de los ecosistemas latinoamericanos.

\section{Páramo y subpáramo}

Los ecosistemas de páramo se encuentran a más de $3600 \mathrm{~m}$ de altitud y los de subpáramo entre 3000 y $3600 \mathrm{~m}$; ambos son ambientes abundantes en Latinoamérica y que son particularmente sensibles al cambio climático. Los páramos y subpáramos, además de ser centros de biodiversidad de especies endémicas, son ambientes que proveen, entre otra serie de bienes y servicios ecosistémicos, agua a las comunidades y fijación de carbono (Anderson et al., 2011). De manera particular, los páramos podrían ser altamente sensibles a los cambios de temperatura (Enquist, 2002), ésto debido a que las especies en los picos de las montañas no tienen adonde migrar altitudinalmente (Buytaert et al., 2011).

Asimismo, han ocurrido períodos de sequía y cambios en la insolación y en la frecuencia e intensidad de la precipitación, dichos cambios no sólo afectan las plantas de páramo, sino que también generan cambios en escorrentía, sedimentación en ríos e incremento en enfermedades provenientes de menores altitudes (Anderson et al., 2011). Al verse afectados estos ecosistemas, se ven perjudicados sus servicios ecosistémicos, por ejemplo, pasar de ser sumideros de carbono a ser emisores de carbono (Buytaert, Cuesta-Camacho \& Tobón, 2011). Ante esto, es imprescindible aumentar la investigación y protección de los sistemas de páramo y subpáramo en Latinoamérica, pues estos ecosistemas latinoamericanos se encuentran dentro de los menos estudiados (Buytaert et al., 2011).

\section{Bosque seco}

El bosque seco es un ambiente tropical adaptado a la sequía estacional; sin embargo, puede encontrarse en umbrales hidrológicos que lo hacen vulnerable ante el cambio climático (Enquist, 2002; Miles et al., 2006). A nivel mundial, los bosques secos tropicales son ambientes altamente amenazados por actividades antropogénicas (Quesada et al., 2009), principalmente por cambios en el uso del suelo. Debido a dichas amenazas el área de bosque seco ha disminuido tanto a nivel mundial como en América Latina y el Caribe. En esta región ya hemos perdido gran parte de estos bosques, mientras que los que quedan se ven afectados por cambios en el uso de suelo, incendios forestales, fragmentación de hábitat, entre muchos otros factores (Miles et al., 2006). Estas amenazas ya presentes agravan aún más la vulnerabilidad de estos ecosistemas ante el cambio climático.

\section{Océanos}

El océano cubre el $70 \%$ de la superficie del planeta e interactúa constantemente con la atmósfera, alterándose el uno al otro mutuamente de cara a cualquier cambio. Al alterar la atmósfera por emisiones de $\mathrm{CO}_{2}$, alteramos los océanos principalmente de tres formas: 1 ) calentamiento del océano, el cual está asociado con expansión térmica e incremento en el nivel del mar (Church \& White, 2006); 2) absorción de $\mathrm{CO}_{2}$ por el océano, la cual lleva a la acidificación oceánica (Doney, Fabry, Feely \& Kleypas, 2009); y 3) pérdida de oxígeno disuelto en el agua marina por aumento de temperatura - desoxigenación (Keeling, Körtzinger \& Gruber, 2010). Asimismo, los organismos marinos se ven afectados por los impactos del cambio climático en los océanos. Resultan comunes las migraciones por cambios de temperatura, las afectaciones en 
comportamiento y fisiología, e inclusive, la muerte de algunos de ellos (Walther et al., 2002; Poloczanska et al., 2013). A su vez, los océanos afectan las zonas costeras, las cuales se ven impactadas por el incremento en el nivel del mar, la erosión e inundaciones (Zhang, Douglas \& Leatherman, 2004).

En los mares de zonas frías la temperatura cambia bruscamente de manera natural, sin embargo, estos cambios han sido empeorados por el cambio climático, lo cual ocasiona variaciones en los patrones de viento e incremento en la temperatura del mar (Hayward, 1997). Tal modificación en la temperatura marina genera consecuencias en la distribución de las especies marinas, muchas de ellas de tipo comercial (Hayward, 1997; Roessig, Woodley, Cech \& Hansen, 2004). Lo anterior afecta a las comunidades pesqueras en general y a los recolectores costeros en particular, pues dependen de los recursos marinos que se ven amenazados por el cambio climático.

Además, hay que mencionar que existen múltiples factores estresantes adicionales en estos ambientes (Roessig et al., 2004). También, a diferencia de los ambientes terrestres, todavía desconocemos mucho sobre la biodiversidad marina, con muchos vacíos de información aún por llenar.

\section{Antártida}

El continente antártico, que se encuentra en el Polo Sur de América, presenta cambios drásticos asociados al cambio climático, los cuales evidencian su vulnerabilidad. De manera natural, la nieve va incrementando la presión hasta crear capas de hielo que luego bajan al mar por ríos congelados, este hielo en condiciones frías no se derrite, con lo que se conforman los glaciares. El cambio climático ha generado un incremento en la temperatura y en los patrones de precipitación en la Antártida (Walther et al., 2002; Turner et al., 2005), lo que ocasiona derretimientos significativos en los glaciares (Rignot, Jacobs, Mouginot \& Scheuchl, 2013) y disminución en la producción de hielo marino (Meredith \& King, 2005). Dichos eventos han ocasionado la colonización y expansión de plantas en zonas terrestres (Walther et al., 2002).

Por su parte, los cambios en la temperatura y la entrada de agua dulce al sistema marino alteran las condiciones del océano y su biota asociada, incluyendo el fitoplancton marino. Por lo general, la biota antártica posee nichos fisiológicos amplios para soportar las condiciones cambiantes en el sitio (Walther et al., 2002). Sin embargo, dicha biota también suele vivir cerca de sus puntos de quiebre de tolerancia, lo cual hace que sea aún más vulnerable a los cambios ocasionados por el cambio climático (Walther et al., 2002). Aún falta mucho por investigar en el continente antártico, pero no hay duda que es un sistema altamente sensible al cambio climático.

\section{MITIGACIÓN DEL CAMBIO CLIMÁTICO}

Ante el incremento en las concentraciones de gases de efecto invernadero en la atmósfera por causas antropogénicas, en particular el dióxido de carbono $\left(\mathrm{CO}_{2}\right)$, tenemos la responsabilidad de bajar dichas concentraciones. A esto se le conoce como mitigación, la cual se puede realizar: 1) al dejar de emitir dichos gases (descarbonización); 2) proteger los reservorios naturales de carbono para evitar su emisión; y 3) activamente extraer $\mathrm{CO}_{2}$ de la atmósfera naturalmente o por mecanismos artificiales.

\section{Sector transporte}

El sector transporte, ya sea terrestre, aéreo o marítimo, sigue siendo el responsable de grandes cantidades de emisiones de gases de efecto invernadero (GEI) tanto en países latinoamericanos (Chacón Araya, Jiménez Valverde, Montenegro Ballestero, Sasa Marín \& Blanco Salas, 2014) como en el resto del mundo (Pachauri et al., 2014). Un motivo de la gran cantidad de emisiones del sector transporte es la limitación en el desarrollo de transporte público descarbonizado en Latinoamérica, donde resulta más llamativo el uso del automóvil particular, principalmente debido a la falta de inversión en el mejoramiento del transporte público.

El transporte urbano sostenible busca mitigar la emisión de GEI al modificar los mecanismos de transporte actual (Pojani \& Stead, 2015). Para dicha reducción en emisiones se considera que es necesario disminuir la cantidad de viajes realizados por medio de un desarrollo urbano mixto condensado, dado que la interconectividad entre los distintos servicios y sectores de las ciudades es clave para un transporte público exitoso. También, es fundamental que existan métodos sostenibles de transporte como el uso de la bicicleta, el caminar y uso del transporte público bajo en emisión, los cuales deben ser confiables, interconectados y seguros. Dado que el uso de automóviles eléctricos es muy limitado, por cuanto el precio es elevado y esto limita la cantidad de personas que los pueden adquirir, para lograr dicha meta de reducción de emisiones, un aspecto crucial es que se mejore la tecnología de transporte existente mediante una priorización del transporte público sostenible (Pojani \& Stead, 2015).

En los diversos países latinoamericanos existen varios ejemplos a seguir en el tema de transporte sostenible; a 
modo de ilustración: los autobuses eléctricos en Santiago y la Red de Ciclovías en Bogotá y en Sao Paulo. El transporte sostenible no solo disminuye los GEl, sino que también mejora la calidad del aire y disminuye tanto el ruido y como los siniestros (Kwan \& Hashim, 2016). Para poder cumplir las metas de descarbonización en el transporte, un reto a superar es que las políticas de transporte sostenible son invasivas, dado que impactan el estilo de vida de todos los habitantes y la congestión vial es un tema muy democrático. Llegar a tener una Latinoamérica con una adecuada implementación del transporte sostenible es indispensable ante los efectos del cambio climático.

\section{Energías limpias}

Más allá del sector transporte, para la mitigación del cambio climático es indispensable el desarrollo y el fomento de energías limpias. Un modelo positivo es el caso de Costa Rica, líder mundial en la temática de uso de energías renovables, entre ellas las procedentes de fuentes de plantas hidroeléctricas, de energía eólica, geotérmica y solar, así como de biomasa. En el año 2016, durante 250 días Costa Rica utilizó, únicamente electricidad de fuentes renovables, por lo tanto, empleó $98 \%$ de energía renovable en el año (Nield, 2017). Del mismo modo lo hizo en el año 2017, cuando empleó energía renovable por 300 días (Dockrill, 2017; Embury-Dennis, 2017). Ahora, si bien es cierto que ha habido un gran desarrollo en economía de energías renovables (Timmons, Harris, \& Roach, 2014), éstas suelen tener un costo alto, además de que son utilizadas por una minoría de personas.

En este sentido, para mejorar la competitividad de las energías limpias, el costo de energías no renovables debería a futuro incluir todos los costos adicionales asociados, incluyendo el impacto al ambiente. Por ejemplo, el precio del combustible debe incluir el precio del efecto del cambio climático y el costo de mitigarlo (Delucchi, \& Jacobson, 2011; Jacobson, \& Delucchi, 2011).

\section{Reservorios de carbono: bosques terrestres}

Los bosques terrestres tienen la capacidad de disminuir la cantidad de GEI de la atmósfera, dado que absorben $\mathrm{CO}_{2}$ y lo retienen en sus tejidos vegetales por períodos de tiempo prolongados. De esta manera, los bosques representan $70-80 \%$ del reservorio de carbono terrestre en biomasa (Kindermann, McCallum \& Fritz, 2008), y los bosques tropicales encabezan la lista, con un $66 \%$ del total global (Van der Werf et al., 2009; Pan, Birdsey, Phillips \& Jackson, 2013). Los suelos tropicales también contienen grandes reservas de carbono las cuales se reducen por actividades antropogénicas como la deforestación, la agricultura intensiva y la quema de turberas de bajura (Scharlemann, Tanner, Hiederer \& Kapos, 2014). Las emisiones de $\mathrm{CO}_{2^{\prime}}$ debido a deforestación y degradación de bosques y quema de turberas, son la segunda fuente antropogénica de carbono a la atmósfera (12-20\% de las emisiones totales) (Van der Werf et al., 2009). Es por eso que evitar la deforestación y la degradación de los bosques tropicales es una de las estrategias prioritarias de la Convención Marco de las Naciones Unidas sobre el cambio climático (CMNUCC) para mitigar el cambio climático, a través de procesos como REDD+ (Reducción de Emisiones por Deforestación y Degradación de los bosques; www.un-redd.org) (Miles \& Kapos, 2008). Es importante considerar la biodiversidad a la hora de implementar actividades REDD+ (Anderson-Teixeira, 2018) ya que las especies vegetales tienen características diferentes que afectan cuánto carbono se captura y por cuanto tiempo (Díaz, Hector \& Wardle, 2009).

\section{ADAPTACIÓN AL CAMBIO CLIMÁTICO}

Ante los impactos actuales y futuros del cambio climático y los retos que tenemos para mitigar efectivamente los gases causantes del cambio climático, es crucial desarrollar procesos de adaptación, esto es, anticipar sus impactos y actuar o tomar decisiones adecuadas para minimizar o eliminar dichos impactos o sus causantes; así como la capacidad de aprovechar oportunidades ventajosas que puedan surgir de estas. La planificación adecuada y a tiempo es una herramienta sumamente útil para que Latinoamérica pueda resistir y recuperarse ante los impactos negativos del cambio climático.

\section{Áreas protegidas}

El cambio climático afecta la biodiversidad de diversas maneras dependiendo de las especies presentes, su papel funcional en el ecosistema, la redundancia de papeles entre especies distintas y sus niveles en la red trófica (Fonseca \& Ganade, 2001; Walther et al., 2002; Thuiller, Lavorel \& Araújo, 2005); así como la velocidad del cambio ocasionado por el cambio climático y las barreras geográficas que impidan el desplazamiento vertical y horizontal de las especies a nuevos sitios (Burrows et al., 2011; Poloczanska et al., 2013; Burrows et al., 2014). Para evitar su extinción, las especies pueden responder ante el cambio climático cambiando su distribución geográfica, al movilizarse a un sitio más favorable o modificando su fisiología a las nuevas condiciones, así como por medio de evolución adaptativa (Hoffmann \& Sgrò, 
2011). Ante la movilización y pérdida de especies debido al cambio climático, las áreas protegidas son una herramienta crucial; así como el fortalecimiento y creación de corredores biológicos y áreas amortiguadores para el desplazamiento de las especies, basados en mapas de vías y velocidades probables de migración de especies (Gillson, Dawson, Jack \& McGeoch, 2013). Para fortalecer la biodiversidad y ecosistemas dentro de las áreas protegidas es crucial minimizar o eliminar los impactos no asociados al cambio climático. También, es importante seleccionar aquellos sitios que contengan sistemas con mayor resiliencia ante el cambio climático y ante especies invasoras (Gillson et al., 2013); por lo que los análisis de vulnerabilidad espacial ante el cambio climático son críticos. Se deben identificar áreas prioritarias para la conservación, restauración ecológica y corredores de migración, así como la conservación ex situ de especies que no tienen adonde migrar (Hoegh-Guldberg et al., 2008). Con el conocimiento actual sobre los impactos del cambio climático a la biodiversidad no se puede predecir cuáles especies dominarán los ambientes en el futuro, pero sí se sabe que hay especies más vulnerables que otras y es claro que se requiere mayor investigación sobre el tema.

\section{Adaptación basada en ecosistemas}

En Latinoamérica gran parte de la población depende directamente de los recursos naturales y los servicios ecosistémicos que proveen los ambientes, los cuales se ven amenazados por el cambio climático. La adaptación basada en ecosistemas (AbE) es una herramienta ante el cambio climático que busca preservar la biodiversidad y ambientes por medio de la integración del componente humano y el desarrollo económico sostenible; es decir, busca la protección de los ambientes para que puedan brindar sus servicios ecosistémicos a la sociedad y ambos sean más resilientes ante el cambio climático (Vignola, Locatelli, Martinez \& Imbach, 2009). La biodiversidad y los servicios que los ecosistemas proveen a las poblaciones humanas son parte de una estrategia integral más amplia de adaptación para ayudar a las personas a enfrentar efectos diversos del cambio climático, con beneficios añadidos de mitigación, protección de medios de subsistencia y disminución de la pobreza (Munang et al., 2013). Actualmente, según la UICN, en Latinoamérica existen múltiples proyectos que buscan fortalecer a las comunidades y los ecosistemas ante el cambio climático.

\section{Agroecología}

Los países latinoamericanos son fuertemente dependientes de los agroecosistemas, tanto para subsistencia como para exportación, los cuales se ven amenazados por el cambio climático, pero pueden ser modificados para mejorar su respuesta. Los agroecosistemas incluyen sistemas de mono y policultivos y sistemas de agricultura mezclada con ganadería, silvicultura y acuacultura (Fuhrer, 2003), incluyendo monocultivos intensivos dependientes de agroquímicos. El cambio climático afecta a estos agroecosistemas principalmente causando una disminución en la productividad y mayor susceptibilidad a plagas y enfermedades, debido a cambios en la temperatura, patrones de precipitación y ciclos de nutrientes (Fuhrer, 2003). Variaciones ambientales asociadas al cambio climático ocasionan grandes pérdidas de cultivos.

La diversificación de los cultivos puede incrementar la resiliencia de estos agrosistemas ante el cambio climático, lo cual les permite responder mejor (Lin, 2011). Existen diversas maneras de diversificar los agrosistemas, a manera de variedad genética, a nivel de especies o estructural, lo cual se puede hacer a escala pequeña dentro de la plantación o hasta nivel de paisaje (Lin, 2011). Tradicionalmente en Latinoamérica los agroecosistemas se han desarrollado en pequeñas parcelas de tierra, con mayor diversidad de cultivos y tipos de producción, en particular la mezcla de sistemas de producción mixta de plantas y animales (Altieri, 1999). Estos sistemas de cultivo más tradicionales son mucho más productivos en su totalidad y más biodiversos que los monocultivos (Altieri, 1999); lo cual los hace más resilientes ante el cambio climático; por lo cual debemos investigar y aplicar más estos agroecosistemas biodiversos tradicionales.

\section{Salud humana}

El cambio climático tiene efectos directos e indirectos sobre la salud humana, un tema que debe ser tratado con más profundidad y urgencia. Los efectos directos incluyen aquellos asociados a olas de calor, sequías, humo de incendios forestales, inundaciones, precipitaciones, huracanes, contaminación del aire y alérgenos; así como enfermedades infecciosas o por vectores (Haines \& Patz, 2004; Moreno, 2006). Los efectos indirectos están relacionados principalmente con la producción de alimentos y alteración de nichos de vectores de enfermedades que migran horizontal y verticalmente a zonas ahora 
más calientes (Haines \& Patz, 2004). También, puede haber mayor impacto de las mareas rojas, por toxinas del fitoplancton en organismos filtradores (Moreno, 2006). En Centroamérica hay incremento en enfermedad renal crónica asociado a estrés hídrico y térmico en trabajadores agrícolas en condiciones de sequía (Weiner, McClean, Kaufman \& Brooks, 2012). Los países del Cono Sur están muy atentos al incremento de dengue en zonas donde antes no había por variaciones en temperatura (Haines \& Patz, 2004). Un factor adicional a considerar es que la vulnerabilidad en salud ante el cambio climático no es homogénea entre la población, dado a factores sociales y ambientales, por lo que se debe tomar en cuenta dicho parámetro en las políticas de salud ante el cambio climático.

\section{INCENTIVANDO LA ACCIÓN ANTE EL CAMBIO CLIMÁTICO}

\section{Gobernanza}

El cambio climático tiene una gobernanza (por ejemplo, distribución del poder) compleja y con múltiples niveles, dado que captura factores políticos, socio-económicos y ambientales, así como la gran cantidad de actores involucrados (Betsill \& Bulkeley, 2006). Por ejemplo, para llegar a acuerdos entre gobiernos para disminuir la emisión de GEl, la posición de los países en las negociaciones depende del nivel de contribución al cambio climático y de afectación por el mismo. El acuerdo de París en la COP21 en el 2015 fue un éxito en este sentido ya que define el compromiso de los gobiernos para tomar acciones de mitigación necesarias para mantener el incremento en la temperatura mundial muy por debajo de $2^{\circ} \mathrm{C}$, en el cual América Latina y el Caribe se unen a dicho acuerdo (Schleussner et al., 2016; UNFCCC, 2018). Un reto para la gobernanza del cambio climático es que el bajar la emisión de GEl involucra costos a corto plazo que varían entre los distintos países y es un reto para los políticos justificar gastos a corto plazo para beneficios a largo plazo, lo cual tiene un impacto en agendas electorales. El hecho que todo el planeta utilice energía renovable en las próximas décadas es posible, pero su principal reto es social y político, no tecnológico o de costo (Delucchi, \& Jacobson, 2011; Jacobson, $\&$ Delucchi, 2011). Ante una gobernanza compleja y la urgencia de la descarbonización mundial, los actores emergentes ante el cambio climático no son los países, sino las grandes ciudades, siendo un movimiento tanto local como global, a la vez estatal y no estatal (Betsill \& Bulkeley, 2006). En las grandes ciudades a nivel mundial se aglomera más del $50 \%$ de la población humana y son fuertes emisores de GEl (Kennedy et al., 2009). El diálogo entre las alcaldías de las grandes ciudades es menos complejo que a nivel de países, y puede discrepar con las políticas del gobierno estatal, por lo que actualmente ya hay compromisos ante el cambio climático de 92 ciudades a nivel mundial (www.c40.org). En la gobernanza ante el cambio climático es crítica la participación de los ciudadanos ya que sus acciones pueden tener fuertes impactos ante el cambio climático.

\section{Participación ciudadana}

Ante los retos para la efectiva toma de decisiones ante el cambio climático, considerando además marcos de gobernanza complejos, la participación ciudadana y su consecuente implementación en la política pública, son factores claves para la acción oportuna (Few, Brown \& Tompkins, 2007). La participación ciudadana, como derecho legítimo, se define como la intervención de la ciudadanía en los procesos de toma de decisiones (Naciones Unidas, 1948), representando así un elemento fundamental de un sistema democrático, inclusivo y representativo, en el que se incluyen un conjunto de acciones (formales o informales), al que las personas acudimos para ser parte del proceso deliberativo, de ejecución, fiscalización, monitoreo, entre otros. Las formas de ejercer ciudadanía, a través de la participación pueden ser muy diversas e incluyen tanto opciones propositivas como contestatarias, con foco en alterar directamente los patrones sistemáticos del comportamiento social o de influir en el proceso de política pública; todas ellas en distintos niveles de profundidad y con distintas posibilidades de incidencia.

Además de reconocer las distintas formas de participación, para poder incidir con mayor éxito en esta época de transición, producto del cambio climático, es necesario trabajar sobre ciertos retos, como robustecer las habilidades ciudadanas para una democracia activa y fortalecer las capacidades del aparato institucional y su cuerpo normativo. La participación debe buscar el bien del colectivo de esta y de las próximas generaciones, por lo que representa una oportunidad para fortalecer tanto la sostenibilidad ambiental, como la justicia social.

\section{Género}

Las mujeres conforman la mitad de la población humana; sin embargo, son más vulnerables ante el cambio climático que los hombres (UN Women Watch, 2009). Esta mayor vulnerabilidad se debe a las brechas en género, particularmente en países con mayor pobreza (Denton, 
2002). La pobreza está entrelazada de una manera compleja con la inequidad de género, en muchos países pobres, por ejemplo, las mujeres son quienes cumplen el rol de acarrear el agua, son altamente dependientes de sus cultivos y la mayor cantidad de agricultoras son mujeres; pero no tienen los mismos derechos que los hombres, ni existe equidad de género en tenencia de tierras (Deere \& León, 2003; UN Women Watch, 2009). Otro factor a considerar en mujeres embarazadas es el aumento en la incidencia de pre-eclampsia por consumo agua salinizada (Khan et al., 2011). Otros ejemplos incluyen la mayor posibilidad de picaduras por mosquitos, vectores de enfermedades, debido a una mayor temperatura corporal durante el embarazo (Lindsay, Ansell, Selman, Cox, Hamilton \& Walraven, 2000). En particular, las mujeres suelen estar por fuera de los procesos de toma de decisiones con respecto al cambio climático (Denton, 2002) Sin embargo, en países donde las brechas de género son menores, y las mujeres tienen mayores niveles de acceso a la educación, la equidad en otros aspectos tiende a aumentar. Es claro que las estrategias ante el cambio climático deben incluir a todos los actores involucrados, particularmente aquellos más vulnerables como las mujeres (Alston, 2014). Ante su mayor vulnerabilidad ante el cambio climático es indispensable incorporar y tener en consideración a la población femenina en la respuesta ante el mismo.

\section{Comunicación y educación del cambio climático}

Los científicos, comunicadores y educadores tienen importantes mensajes que transmitir en aras de incentivar acciones adecuadas ante el cambio climático; sin embargo, en muchas ocasiones, las estrategias no son las más apropiadas. La gran cantidad de investigación científica asociada y la compleja gobernanza del cambio climático han presentado un reto para su comunicación efectiva. Un reto en particular es la comunicación entre los científicos, los políticos y la comunidad en general. La ciencia del cambio climático se ha consolidado cada vez más y hay amplia evidencia (Pachauri et al., 2014), se han desarrollado múltiples documentos para análisis y simplificación de dicha ciencia para la comunicación por parte del Grupo Intergubernamental sobre cambio climático (IPCC) y se ha trabajado en comunicar el consenso que existe entre científicos de que el cambio climático es real y es causado por actividades humanas (Cook et al., 2016). Esta información y consenso científico de la severidad y urgencia de acción ante el cambio climático muchas veces no ha sido traducida de manera efectiva a políticas públicas, y tampoco ha sido suficiente para modificar las percepciones que la comunidad latinoamericana, en general, tiene frente al cambio climático. Entre los retos en la comunicación efectiva del cambio climático está el hecho de que los científicos publican sus hallazgos en revistas científicas con terminología compleja, que son leídas principalmente dentro del gremio académico y que incluyen conceptos de variabilidad e incertidumbre que suelen ser mal interpretados por el público en general o los tomadores de decisiones (Somerville \& Hassol, 2011). Otro reto para la comunicación del cambio climático es que es un proceso intangible, en ocasiones con grandes distancias espaciales y temporales entre las causas y los efectos y el que gran parte de la población ya no interactúa constantemente con el ambiente natural (Moser, 2010). A la vez, la comunidad en general está inundada de mensajes contradictorios y en muchas ocasiones alarmistas, en particular a través del internet, donde muchos científicos no tienen altos niveles de participación (Schäfer, 2012). La comunicación efectiva de la ciencia es una herramienta crítica que se debe desarrollar más con relación al cambio climático.

Para una mejor comunicación del cambio climático se recomienda mejorar la narrativa, lo cual incluye: 1) tener claro cuál es el propósito y alcance de lo que se quiere comunicar, 2) cuál es el público; 3) quiénes son los mensajeros; 4) tener claro por qué debería de importarles lo que se está comunicando; 5) palabras más sencillas; 6 ) uso de metáforas o comparaciones con realidades cotidianas; así como 7) el uso de pasión e historias para conectar con el público, no sólo datos (Somerville \& Hassol, 2011).

\section{CONCLUSIÓN}

El cambio climático es real y es causado por actividades humanas, lo cual nos ofrece una oportunidad de cambiar nuestras acciones para remediarlo. El reto es grande y complejo pero entre todos podemos lograrlo. El I Simposio sobre cambio climático y Biodiversidad, en Costa Rica, nos permitió analizar los diversos temas e interactuar entre los diversos actores ante el cambio climático en la región latinoamericana. Queda claro que necesitamos una planificación adecuada y acciones inmediatas en cada uno de los distintos sectores. Estas deben ser inclusivas para todas las personas sin importar su condición socioeconómica, género ni edad. Tenemos muchos retos por superar, desde vacíos en investigación científica en relación al cambio climático hasta cómo comunicar y educar a la comunidad y las próximas generaciones. El cambio climático es un fenómeno complejo, que involucra no sólo a la naturaleza sino todo un conjunto de actores y factores socio-político-económicos. No actuar 
es en sí una acción, la cual tendría consecuencias serias para nuestra generación y las generaciones futuras.

La ciencia de calidad debe ser base indispensable para la toma de decisiones y se debe investigar los temas de mayor necesidad ante el cambio climático, pero debe estar acompañada de un cambio social e inclusive económico, resaltando el papel de la ciencia social en relación al cambio climático. Por tanto, debemos actuar en conjunto, de manera inmediata y teniendo clara la realidad que queremos alcanzar a corto, mediano y largo plazo. No se puede solucionar el problema con la misma mentalidad que se ha tenido hasta ahora; debemos buscar un nuevo camino, para lo cual es importante reconsiderar el modelo socio-económico que ha predominado, así como la forma de hacer política teniendo como consideración clave sus efectos sobre la sociedad y el ambiente.

\section{REFERENCIAS}

Alston, M. (2014). Gender mainstreaming and climate change. Women's Studies International Forum, 47, 287-294.

Altieri, M. A. (1999). Applying agroecology to enhance the productivity of peasant farming systems in Latin America. Environment, Development and Sustainability, 1(3-4), 197-217.

Anderson, E. P., Marengo, J., Villalba, R., Halloy, S., Young, B., Cordero, D., Gast, F., Jaimes, E., Ruiz, D., \& Herzog, S. K. (2011). Consequences of climate change for ecosystems and ecosystem services in the tropical Andes. In S. K. Herzog, R. Martinez, P. M. Jorgensen, \& H. Tiessen (Eds.), Climate change effects on the biodiversity of the tropical Andes: An assessment of the status of scientific knowledge. San Jose dos Campos and Paris: Inter-American Institute of Global Change Research (IAI) and Scientific Committee on Problems of the Environment (SCOPE).

Anderson-Teixeira, K. J. (2018). Prioritizing biodiversity and carbon. Nature Climate Change, 1.

Betsill, M. M., \& Bulkeley, H. (2006). Cities and the multilevel governance of global climate change. Global Governance: A Review of Multilateralism and International Organizations, 12(2), 141-159.

Burrows, M. T., Schoeman, D. S., Buckley, L. B., Moore, P., Poloczanska, E. S., Brander, K. M., Brown, C., Bruno, J. F., Duarte, C. M., Halpern, B. S., Holding, J., Kappel, C. V., Kiessling, W., O'Connor, M. I., Pandolfi, J. M., Parmesan, C., Schwing, F. B., Sydeman, W. J., \& Richardson, A. J. (2011). The pace of shifting climate in marine and terrestrial ecosystems. Science, 334(6056), 652-655.

Burrows, M. T., Schoeman, D. S., Richardson, A. J., Molinos, J. G., Hoffmann, A., Buckley, L. B., Moore, P. J., Brown, C. J., Bruno, J. F., Duarte, C. M., Halpern, B. S., Hoegh-Guldberg, O., Kappel, C. V., Kiessling, W., O’Connor, M. I., Pandolfi, J. M., Parmesan, C., Sydeman, W. J., Ferrier, S., Williams,
K. J., \& Poloczanska, E. S. (2014). Geographical limits to species-range shifts are suggested by climate velocity. Nature, 507(7493), 492.

Buytaert, W., Cuesta-Camacho, F., \& Tobón, C. (2011). Potential impacts of climate change on the environmental services of humid tropical alpine regions. Global Ecology and Biogeography, 20(1), 19-33.

Chacón Araya, A. R., Jiménez Valverde, G., Montenegro Ballestero, J., Sasa Marín, J., \& Blanco Salas, K. (2014). Inventario Nacional de Gases de Efecto Invernadero y Absorción de Carbono 2010: Costa Rica. San José, Costa Rica.

Church, J. A., \& White, N. J. (2006). A 20th century acceleration in global sea-level rise. Geophysical research letters, 33(1).

Cook, J., Oreskes, N., Doran, P. T., Anderegg, W. R., Verheggen, B., Maibach, E. W., Carlton, J. S., Lewandowsky, S., Skuce, A. G., \& Green, S. A. (2016). Consensus on consensus: a synthesis of consensus estimates on human-caused global warming. Environmental Research Letters, 11(4), 048002.

Deere, C. D., \& León, M. (2003). The gender asset gap: land in Latin America. World Development, 31(6), 925-947.

Denton, F. (2002). Climate change vulnerability, impacts, and adaptation: Why does gender matter? Gender \& Development, 10(2), 10-20.

Díaz, S., Hector, A., \& Wardle, D. A. (2009). Biodiversity in forest carbon sequestration initiatives: not just a side benefit. Current Opinion in Environmental Sustainability, 1(1), 55-60.

Doney, S. C., Fabry, V. J., Feely, R. A., \& Kleypas, J. A. (2009). Ocean acidification: the other $\mathrm{CO} 2$ problem. Annual Review of Marine Science, 1, 169-192.

Enquist, C. A. F. (2002). Predicted regional impacts of climate change on the geographical distribution and diversity of tropical forests in Costa Rica. Journal of Biogeography, 29(4), 519-534.

Few, R., Brown, K., \& Tompkins, E. L. (2007). Public participation and climate change adaptation: avoiding the illusion of inclusion. Climate Policy, 7(1), 46-59.

Fonseca, C. R., \& Ganade, G. (2001). Species functional redundancy, random extinctions and the stability of ecosystems. Journal of Ecology, 89(1), 118-125.

Fuhrer, J. (2003). Agroecosystem responses to combinations of elevated $\mathrm{CO} 2$, ozone, and global climate change. Agriculture, Ecosystems \& Environment, 97(1-3), 1-20.

Galindo, L. M., de Miguel, C., \& Ferrer, J. (2010). Gráficos vitales del cambio climático para América Latina y El Caribe: Edición especial para la CP16/CP-RP 6, México: PNUMA; CEPAL; PNUMA/GRID-Arendal.

Gillson, L., Dawson, T. P., Jack, S., \& McGeoch, M. A. (2013). Accommodating climate change contingencies in conservation strategy. Trends in Ecology \& Evolution, 28(3), 135-142. 
Haines, A., \& Patz, J. A. (2004). Health effects of climate change. Jama, 291(1), 99-103.

Hayward, T. L. (1997). Pacific Ocean climate change: atmospheric forcing, ocean circulation and ecosystem response. Trends in ecology \& evolution, 12(4), 150-154.

Hoegh-Guldberg, O., Hughes, L., Mclntyre, S., Lindenmayer, D., Parmesan, C., Possingham, H. P., \& Thomas, C. (2008). Assisted colonization and rapid climate change. Science, 321, 345-346.

Hoffmann, A. A., \& Sgrò, C. M. (2011). Climate change and evolutionary adaptation. Nature, 470(7335), 479-485.

Keeling, R. F., Körtzinger, A., \& Gruber, N. (2010). Ocean deoxygenation in a warming world. Annual Review of Marine Science, 2(463-493).

Kennedy, C., Steinberger, J., Gasson, B., Hansen, Y., Hillman, T., Havranek, M., Pataki, D., Phdungsilp, A., Ramaswami, A., \& Mendez, G. V. (2009). Greenhouse gas emissions from global cities: ACS Publications.

Khan, A. E., Ireson, A., Kovats, S., Mojumder, S. K., Khusru, A., Rahman, A., \& Vineis, P. (2011). Drinking water salinity and maternal health in coastal Bangladesh: implications of climate change. Environmental Health Perspectives, 119(9), 1328.

Kindermann GE, McCallum I, Fritz S, \& M., O. (2008). A global forest growing stock, biomass and carbon map based on FAO statistics. Silva Fennica 42, 387-396.

Kwan, S. C., \& Hashim, J. H. (2016). A review on co-benefits of mass public transportation in climate change mitigation. Sustainable Cities and Society, 22, 11-18.

Lin, B. B. (2011). Resilience in agriculture through crop diversification: adaptive management for environmental change. BioScience, 61(3), 183-193.

Lindsay, S., Ansell, J., Selman, C., Cox, V., Hamilton, K., \& Walraven, G. (2000). Effect of pregnancy on exposure to malaria mosquitoes. The Lancet, 355(9219), 1972.

Meredith, M. P., \& King, J. C. (2005). Rapid climate change in the ocean west of the Antarctic Peninsula during the second half of the 20th century. Geophysical Research Letters, 32(19).

Miles, L., \& Kapos, V. (2008). Reducing greenhouse gas emissions from deforestation and forest degradation: global land-use implications. Science, 320(5882), 1454-1455.

Miles, L., Newton, A. C., DeFries, R. S., Ravilious, C., May, I., Blyth, S., Kapos, V., \& Gordon, J. E. (2006). A global overview of the conservation status of tropical dry forests. Journal of Biogeography, 33(3), 491-505.

Moreno, A. R. (2006). Climate change and human health in Latin America: drivers, effects, and policies. Regional Environmental Change, 6(3), 157-164.

Moser, S. C. (2010). Communicating climate change: history, challenges, process and future directions. Wiley Interdisciplinary Reviews: Climate Change, 1(1), 31-53.
Munang, R., Thiaw, I., Alverson, K., Mumba, M., Liu, J., \& Rivington, M. (2013). Climate change and Ecosystembased Adaptation: a new pragmatic approach to buffering climate change impacts. Current Opinion in Environmental Sustainability, 5(1), 67-71.

Naciones Unidas. (1948). Declaración Universal de los Derechos Humanos. La Convención Internacional de los Derechos del Niño. Declaración sobre la Protección de todas las personas contra la tortura.

Pachauri, R. K., Allen, M. R., Barros, V. R., Broome, J., Cramer, W., Christ, R., Church, J. A., Clarke, L., Dahe, Q., \& Dasgupta, P. (2014). Climate Change 2014: Synthesis Report. Contribution of Working Groups I, II and III to the fifth assessment report of the Intergovernmental Panel on Climate Change: IPCC.

Pan, Y., Birdsey, R. A., Phillips, O. L., \& Jackson, R. B. (2013). The structure, distribution, and biomass of the world's forests. Annual Review of Ecology, Evolution, and Systematics, 44(593-622).

Pojani, D., \& Stead, D. (2015). Sustainable urban transport in the developing world: beyond megacities. Sustainability, 7(6), 7784-7805.

Poloczanska, E. S., Brown, C. J., Sydeman, W. J., Kiessling, W., Schoeman, D. S., Moore, P. J., Brander, K., Bruno, J. F. Buckley, L. B., Burrows, M. T., Duarte, C. M., Halpern, B. S., Holding, J., Kappel, C. V., O'Connor, M. I., Pandolfi, J. M., Parmesan, C., Schwing, F., Thompson, S. A., \& Richardson, A. J. (2013). Global imprint of climate change on marine life. Nature Climate Change, 3(10), 919.

Quesada, M., Sanchez-Azofeifa, G. A., Alvarez-Anorve, M., Stoner, K. E., Avila-Cabadilla, L., Calvo-Alvarado, J., Castillo, A., Espírito-Santo, M. M., Fagundes, M., Fernandes, G. W., Gamon, J., Lopezaraiza-Mikel, M., Lawrence, D., Cerdeira Morellato, L. P., Powers, J., Neves, F. d. S., Rosas-Guerrero, V., Sayago, R., \& Sanchez-Montoya, G. (2009). Succession and management of tropical dry forests in the Americas: Review and new perspectives. Forest Ecology and Management, 258(6), 1014-1024.

Ready, P. (2008). Leishmaniasis emergence and climate change. Revue Scientifique et Technique , 27(2), 399-412.

Rignot, E., Jacobs, S., Mouginot, J., \& Scheuchl, B. (2013). Iceshelf melting around Antarctica. Science, 341(6143), 266-270.

Roessig, J. M., Woodley, C. M., Cech, J. J., \& Hansen, L. J. (2004). Effects of global climate change on marine and estuarine fishes and fisheries. Reviews in Fish Biology and Fisheries, 14(2), 251-275.

Schäfer, M. S. (2012). Online communication on climate change and climate politics: a literature review. Wiley Interdisciplinary Reviews: Climate Change, 3(6), 527-543.

Scharlemann, J. P., Tanner, E. V., Hiederer, R., \& Kapos, V. (2014). Global soil carbon: understanding and managing the largest terrestrial carbon pool. Carbon Management, 5(1), 81-91. 
Schleussner, C.-F., Rogelj, J., Schaeffer, M., Lissner, T., Licker, R., Fischer, E. M., Knutti, R., Levermann, A., Frieler, K., \& Hare, W. (2016). Science and policy characteristics of the Paris Agreement temperature goal. Nature Climate Change, 6(9), 827.

Somerville, R. C., \& Hassol, S. J. (2011). Communicating the science of climate change. Physics Today, 64(10), 48-53.

Thuiller, W., Lavorel, S., \& Araújo, M. B. (2005). Niche properties and geographical extent as predictors of species sensitivity to climate change. Global Ecology and Biogeography, 14(4), 347-357.

Turner, J., Colwell, S. R., Marshall, G. J., Lachlan-Cope, T. A., Carleton, A. M., Jones, P. D., Lagun, V., Reid, P. A., \& lagovkina, S. (2005). Antarctic climate change during the last 50 years. International Journal of Climatology, 25(3), 279-294.

UN Women Watch. (2009). Women, Gender Equality and Climate Change. Retrieved from http://www.un.org/ womenwatch/feature/climate_change/

UNFCCC. (2018). Paris agreement: status of ratification. Retrieved from http://unfccc.int/paris_agreement/ items/9444.php

Van der Werf, G. R., Morton, D. C., DeFries, R. S., Olivier, J. G., Kasibhatla, P. S., Jackson, R. B., Collatz, G. J., \& Randerson,
J. T. (2009). CO2 emissions from forest loss. Nature Geoscience, 2(11), 737.

Vignola, R., Locatelli, B., Martinez, C., \& Imbach, P. (2009). Ecosystem-based adaptation to climate change: what role for policy-makers, society and scientists? Mitigation and Adaptation Strategies for Global Change, 14(8), 691.

Waters, C. N., Zalasiewicz, J., Summerhayes, C., Barnosky, A. D., Poirier, C., Gałuszka, A., Cearreta, A., Edgeworth, M., Ellis, E. C., Ellis, M., Jeandel, C., Leinfelder, R., McNeill, J. R., Richter, D. d., Steffen, W., Syvitski, J., Vidas, D., Wagreich, M., Williams, M., Zhisheng, A., Grinevald, J., Odada, E., Oreskes, N., \& Wolfe, A. P. (2016). The Anthropocene is functionally and stratigraphically distinct from the Holocene. Science, 351(6269), aad2622.

Walther, G.-R., Post, E., Convey, P., Menzel, A., Parmesan, C., Beebee, T. J., Fromentin, J.-M., Hoegh-Guldberg, O., \& Bairlein, F. (2002). Ecological responses to recent climate change. Nature, 416(6879), 389.

Weiner, D. E., McClean, M. D., Kaufman, J. S., \& Brooks, D. R. (2012). The central American epidemic of CKD. Clinical Journal of the American Society of Nephrology, CJN. 05050512.

Zhang, K., Douglas, B. C., \& Leatherman, S. P. (2004). Global warming and coastal erosion. Climatic Change, 64(1-2), 41. 